\title{
Estructura poblacional de Mauritia flexuosa y Oenocarpus bataua en tres comunidades de la cuenca alta del Putumayo, frontera Perú - Colombia
}

\author{
[Population structure of Mauritia flexuosa and Oenocarpus bataua in three \\ communities of the upper Putumayo river basin, Peru - Colombia border]
}

\author{
Luis Freitas Alvarado*1, Valentín Dávila Macedo², Pedro Pérez Peña², Roberto Pezo Díaz³, \\ Kember Mejía²
}

1. Instituto de Investigaciones de la Amazonía Peruana (IIAP). Dirección de Investigación en Manejo Integral del Bosque y Servicios Ecosistémicos (BOSQUES). Av. A. Quiñones km 2,5, San Juan Bautista, Maynas, Loreto, Perú.

Correo electrónico: Ifreitas@iiap.gob.pe (L. Freitas * Autor para correspondencia).

2. Instituto de Investigaciones de la Amazonía Peruana (IIAP). Dirección de Investigación en Diversidad Biológica

Terrestre Amazónica (DBIO). Av. A. Quiñones km 2,5, San Juan Bautista, Maynas, Loreto, Perú. Correos electrónicos: devadama96@gmail.com (V. Dávila), pperez@iiap.gob.pe (P. Pérez), kmejia@iiap.gob.pe (K. Mejía).

3. Universidad Nacional de la Amazonía Peruana (UNAP). Facultad de Ciencias Biológicas (FCB). Campus Universitario

Zungarococha, San Juan Bautista, Loreto, Perú. Correo electrónico: roberto.pezo.246@unapiquitos.edu.pe (R. Pezo).

\section{Resumen}

Las palmeras Mauritia flexuosa y Oenocarpus bataua son muy utilizadas por las comunidades ribereñas pero son muy poco estudiadas y amenazadas por su aprovechamiento inadecuado. Por lo cual, el objetivo fue proveer información sobre la estructura poblacional del aguaje (Mauritia flexuosa) y ungurahui (Oenocarpus bataua), con la finalidad de plantear recomendaciones de manejo sostenible. Se realizaron evaluaciones en parcelas estructurales de 1 ha con subparcelas de $10 \mathrm{~m} \times 10 \mathrm{~m}$ anidadas en los extremos. M. flexuosa presentó mayor densidad en Nuevo Belén con 67,8 ind./ha; O. bataua presentó mayor densidad en Nueva Jerusalén con 20 ind./ha. M. flexuosa presentó mayor porcentaje de adultos masculinos con $67 \%$ y $69 \%$ en las dos áreas, la producción de racimos fue mayor en Nuevo Belén con 174 racimos/ha con una producción estimada de 130 sacos equivalente a unas $5 \mathrm{t}$. $O$. bataua presentó mayor producción de racimos en Nueva Jerusalén con 24 racimos/ha y una producción estimada de 12 sacos. La baja densidad de plantas adultas, el ratio del sexo, las distribuciones diamétricas y por altura indican que ambas zonas han sido aprovechadas. Se sugiere que es necesario implementar campañas de educación ambiental orientadas al manejo sostenible de estas especies.

Palabras clave: Arecaceae, Evaluación de palmeras, Fenología, Frutos, Humedales.

\begin{abstract}
The Mauritia flexuosa and Oenocarpus bataua palm trees are widely used by riverside communities. Thorough studies are lacking and threat and existing wild stands are threatened by poor harvesting practices. The objective of this study was to provide information on the population structures of $M$. flexuosa and $O$. bataua, in order to make recommendations for its sustainable management. Evaluations were carried out in structural plots of 1 ha with subplots of $10 \mathrm{~m} \times 10 \mathrm{~m}$ nested at the extremities. M flexuosa presented higher density in Nuevo Belen with 67,8 ind./ha; $O$. bataua presented higher density in Nueva Jerusalem with 20 ind./ha. M. flexuosa presented a higher percentage of male adults with $67 \%$ and $69 \%$ in the two areas, fruiting raceme production was higher in Nuevo Belén with 174 bunches / ha with an estimated production of 130 bags equivalent to about 5 t. $O$. bataua presented the highest bunch production in Nueva Jerusalem with 24 bunches / ha and an estimated production of 12 bags. The results of low density of adult plants, the sex ratio, the diameter and height distributions indicate that both zones have been exploited. It is necessary to implement environmental education campaigns aimed at the sustainable management of these species.
\end{abstract}

Keywords: Arecaceae, Fruits, Palm survey, Phenology, Wetland. 


\section{INTRODUCCIÓN}

Las palmas constituyen el grupo de plantas útiles más importante en los bosques de la Amazonía para las poblaciones rurales y son un elemento destacado, tanto por su diversidad como por su abundancia en todos los hábitats (Parodi y Freitas, 1990; Gilmore et al., 2013; Balslev et al., 2008). Entre las especies de palmas más importantes podemos mencionar a Mauritia flexuosa conocida comúnmente como aguaje en Perú, Buriti en Brasil y cananguche o moriche en Colombia; y Oenocarpus bataua conocida como ungurahui en Perú, patahuá en Brasil y mil pesos en Colombia. El aguaje se desarrolla en zonas pantanosas, permanentemente inundados y mal drenados, denominadas comúnmente aguajales en Perú (Neiff et al., 2004; Encarnación, 1993). El ungurahui se desarrolla y predomina naturalmente en ecosistemas forestales de suelos de poco drenaje como de terraza alta o baja (Honorio et al., 2008; y Montúfar et al., 2010), denominados ungurahuales en Perú (Moreira y Colombier, 2019), pero también se encuentra asociado con el aguaje (Zarate et al. 2019).

La extensión de los ecosistemas de aguajal en la Amazonía peruana supera los cinco millones de hectáreas (OSINFOR, 2011). Los aguajales pueden ser mixtos cuando forman asociaciones con otras especies de manera homogénea sin perder la denominación de aguajal. Además hay aguajales densos con más de 250 individuos adultos por hectárea (Malleaux, 1973; Encarnación, 1993). La información sobre ungurahuales es escaza, existen estudios de tipo social en comunidades indígenas (Sosnowska et al., 2010), evaluaciones del recurso para conocer su abundancia, relación de la altura y el diámetro con la productividad de frutos (Góngora, 2018; Dávila, 2015).

El aguaje y ungurahui, forman parte importante de la dieta alimenticia de los pobladores Amazónicos por sus frutos deliciosos y nutritivos, además son también fuente de alimento para muchos de los animales que se cazan en el bosque y estos a su vez cumplen un papel de dispersión y depredación de las mismas (Bodmer, 1991; Stevenson, 2000; Stevenson et al., 2002; Acevedo y Zamora, 2016). Adicionalmente cumplen funciones ecológicas importantes, los aguajales constituyen grandes almacenes de carbono principalmente debajo del suelo (Lähteenoja y Page, 2011; Freitas et al., 2006; Goodman et al., 2013; Draper et al., 2014).

Tradicionalmente, el aprovechamiento de las especies de palmeras ha estado basado en el simple extractivismo, cortando la palmera para cosechar los frutos (Vormisto, 2002; Hergoualc'h et al., 2017; Horna y Coronado, 2018). En muchos casos, la presión sobre los recursos en la Amazonía va en aumento, como resultado de la creciente demanda generada por el incremento poblacional (Jarrín et al., 2017); así, el consumo anual de aguaje en la ciudad de Iquitos, capital de la región Loreto, es de 230000 sacos que equivalen a 8206 TM (Horn et al., 2018), estimándose que para abastecer este volumen de frutos se cortan unas 115000 palmeras, son pocos los pobladores rurales que utilizan técnica de manejo sostenible para el aprovechamiento de los frutos. No se cuenta con estadísticas de consumo de ungurahui, pero la disminución de abastecimiento es bastante crítica, tal como se observa en la ciudad de Iquitos.

La cuenca alta del río Putumayo es hogar de muchas comunidades indígenas secoyas y kichwas, sociedades que escasamente son tomadas en cuenta en las políticas de desarroIlo; esta zona, alberga alta diversidad de flora y fauna. Estudios recientes realizados en esta zona demostraron que los animales de caza se están cazando de manera sostenible, aunque los primates grandes tienen densidades bajas, por ello es necesario seguir potenciando el uso sostenible (Ramos-Rodriguez et al., 2019); en este mismo sentido las palmeras de aguaje $y$ ungurahui cerca de sus comunidades son taladas para su aprovechamiento (Zárate et al., 2019). Es decir, el aprovechamiento de las palmeras se está realizando de forma inapropiada que dentro de poco afectará a la fauna silvestre (Bodmer et al., 1999). Es por ello que conocer 
el estado actual de estos ecosistemas ayudará a plantear pautas para su aprovechamiento y manejo sostenible.

El aguaje es una palma que en su medio natural puede alcanzar hasta $35 \mathrm{~m}$ de altura, pertenece a la familia Arecaceae, presenta un tallo que puede alcanzar $50 \mathrm{~cm}$ de diámetro en estado adulto, hojas redondas y palmeadas. Se desarrolla en forma gregaria en comunidades boscosas denominadas aguajales, en zonas permanentemente inundables formando superficies monoespecíficas. El sistema sexual del aguaje esta poco estudiado, sin embargo algunos autores mencionan que es una especie polígama es decir produce plantas femeninas, masculinas o hermafroditas (Listabarth, 1999; Freitas et al., 2006). La producción promedio de cosecha de una palma puede alcanzar en promedio $71 \mathrm{ki}-$ los de frutos al año. Desempeña un papel muy importante dentro de la cadena trófica del bosque, pues constituye el principal alimento de mamíferos y otros vertebrados (Aquino, 2005; Beck, 2006; Van der Hoek et al., 2019; Gilmore et al., 2013).

El ungurahui es una palma que pertenece a la familia Arecaceae, puede alcanzar una altura de $22 \mathrm{~m}$, con hojas muy largas y divididas, y tallo que puede alcanzar los $25 \mathrm{~cm}$ de diámetro. Inflorescencia en forma de cola de caballo, de color amarillo en flor y negro-violáceo en fruto. Distribuido en tierras bajas en la Amazonía y crece en bosques de tierra firme. La densidad de palmas varía de 15 a 160 ind./ha. Es una especie monoica, con flores masculinas y femeninas en la misma inflorescencia (Bernal y Galeano, 2013).

La información poblacional es clave en el manejo sostenible del recurso porque permite crear cuotas de aprovechamiento, es por ello que el objetivo del estudio es generar información poblacional sobre las palmeras de aguaje y ungurahui con la finalidad de plantear alternativas de manejo sostenible.

\section{MATERIALES Y MÉTODO}

\section{Área de estudio}

La zona de estudio se localiza en la cuenca alta del río Putumayo, frontera Perú - Colombia, la investigación se desarrolló en tres comunidades indígenas: Nueva Jerusalén, Mashunta y Nuevo Belén, pertenecientes a las etnias Secoyas y Kichwas (Figura 1), las coordenadas geográficas se indican en la Tabla 1. El acceso a las comunidades puede hacerse por vía fluvial, desde la localidad de San Antonio del Estrecho, capital de la provincia del Putumayo con una duración de 10 horas y por vía aérea desde la ciudad de Iquitos con una duración de 1 hora con 50 minutos.

Según la posición geográfica del área de estudio, corresponde a un tipo de clima ecuatorial, se caracteriza por presentar precipitaciones significativas que varían en un rango de $1180 \mathrm{~mm}$ y $3469 \mathrm{~mm}$. La temperatura media mensual en el área varía de $25,9^{\circ} \mathrm{C}$ a $27,08^{\circ} \mathrm{C}$ con una variación térmica de $1,18^{\circ} \mathrm{C}$. En la estación más cercana a la zona de estudio San Antonio del Estrecho es de $25,9^{\circ} \mathrm{C}$. En la comunidad de San Antonio del Estrecho la precipitación promedio mensual es de $2646 \mathrm{~mm}$. La humedad relativa promedio anual de $88,6 \%$ (PEDICP, 2005).

\section{Inventario de aguajales y ungurahua- les}

La unidad de muestreo está conformada por una parcela de 1 ha y dos parcelas anidadas de $10 \times 10$ m ubicadas en dos vértices opuestos de la parcela. En la parcela de 1 ha se registró información de la vegetación mayor que comprende individuos con diámetro $\geq 10 \mathrm{~cm}$ y $\geq 6$ $\mathrm{m}$ de altura total (considerada como grupo de plantas adultas). En las subparcelas de $10 \times 10$ $\mathrm{m}$, se levantó información según la categoría de regeneración o tamaño de planta: 1) brinzales, plantas de 0,3 m - 1,5 m de altura; 2) latizales, plantas de 1,5 m - $6 \mathrm{~m}$ de altura. Se establecieron en total 17 parcelas de muestreo: para aguaje, 3 en Nueva Jerusalén y 5 en Nuevo Belén; para ungurahui, 3 en Nueva Jerusalén y 6 en Mashunta (Tabla 1, Figura 1). El estudio 
de campo fue desarrollado durante el mes de setiembre del año 2019.

\section{Información registrada}

Para el grupo de plantas con fuste $\geq 10 \mathrm{~cm}$ de diámetro a la altura del pecho (DAP) y $\geq 6 \mathrm{~m}$ de altura, la información registrada fue la siguiente: altura total $(m)$, altura del estípite $(m)$, sexo (masculino, femenino o hermafrodita) solamente para el aguaje), estado fenológico (espádice, inflorescencia e infrutescencia) y número de racimos. Para los individuos muestreados en las subparcelas de $10 \times 10 \mathrm{~m}$ se hizo un conteo de plántulas $\leq 0,30 \mathrm{~m}$, mientras que para el grupo de plantas $\geq 0,3 \mathrm{~m}-1,5 \mathrm{~m}$ y $1,5-6,0 \mathrm{~m}$ se realizaron mediciones de la altura.

En el aguaje, se consideró como palmera adulta cuando el individuo desarrolló uno o más racimos con flores, frutos, o ambos; el cual ayudó a

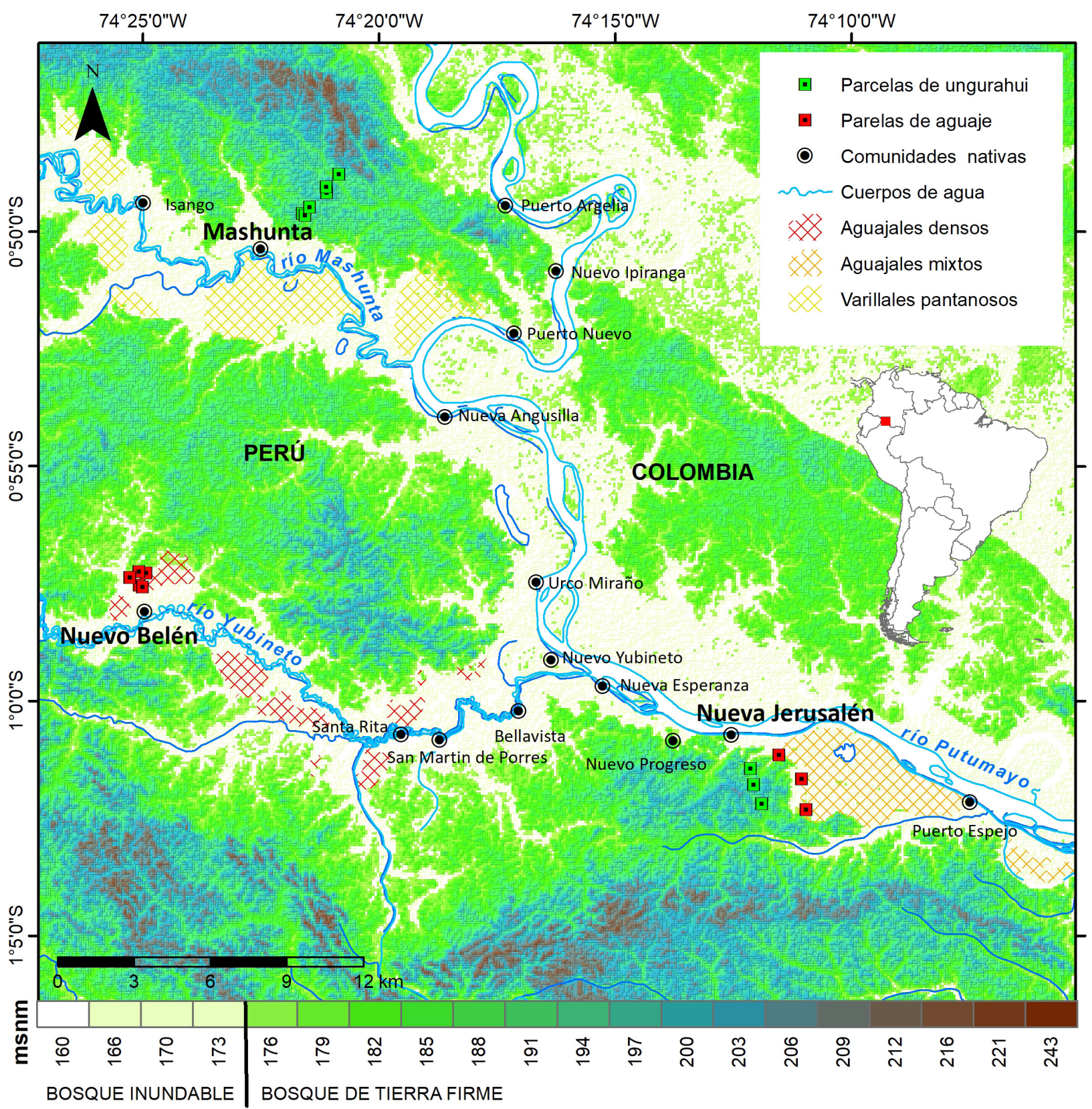

Figura 1. Mapa de la zona de estudio mostrando las parcelas de muestreo. Nótese que la coloración más pálida corresponde al bosque inundable y la coloración más viva al bosque de tierra firme. Los aguajales están en el bosque inundable y los ungurahuales en tierra firme. Las coberturas de aguajales y varillales pantanosos se obtuvieron de Zarate et al. (2019). 
Tabla 1. Coordenadas de las parcelas de muestreo de aguaje y ungurahui en las tres zonas de estudio que pertenece a la zona UTM 18, Loreto, Perú.

\begin{tabular}{|c|c|c|c|c|}
\hline Zona & $\mathbf{N}^{\circ}$ de Parcela & Vértice & $\mathbf{X}$ & $\mathbf{Y}$ \\
\hline \multirow{24}{*}{ Nueva Jerusalén } & 1 & 1 & 589817 & 9887387 \\
\hline & 1 & 2 & 589916 & 9887308 \\
\hline & 1 & 3 & 589913 & 9887394 \\
\hline & 1 & 4 & 589836 & 9887293 \\
\hline & 2 & 1 & 590739 & 9886458 \\
\hline & 2 & 2 & 590795 & 9886439 \\
\hline & 2 & 3 & 590806 & 9886345 \\
\hline & 2 & 4 & 590707 & 9886356 \\
\hline & 3 & 1 & 590868 & 9885236 \\
\hline & 3 & 2 & 590984 & 9885161 \\
\hline & 3 & 3 & 590964 & 9885252 \\
\hline & 3 & 4 & 590890 & 9885141 \\
\hline & 1 & 1 & 588706 & 9886856 \\
\hline & 1 & 2 & 588795 & 9886768 \\
\hline & 1 & 3 & 588764 & 9886849 \\
\hline & 1 & 4 & 588707 & 9886746 \\
\hline & 2 & 1 & 588906 & 9886241 \\
\hline & 2 & 2 & 588851 & 9886117 \\
\hline & 2 & 3 & 588811 & 9886195 \\
\hline & 2 & 4 & 588936 & 9886152 \\
\hline & 3 & 1 & 589249 & 9885451 \\
\hline & 3 & 2 & 589244 & 9885370 \\
\hline & 3 & 3 & 589140 & 9885401 \\
\hline & 3 & 4 & 589144 & 9885485 \\
\hline \multirow{24}{*}{ Mashunta } & 1 & 1 & 571189 & 9908535 \\
\hline & 1 & 2 & 571128 & 9908645 \\
\hline & 1 & 3 & 571219 & 9908628 \\
\hline & 1 & 4 & 571123 & 9908548 \\
\hline & 2 & 1 & 571194 & 9908494 \\
\hline & 2 & 2 & 571330 & 9908542 \\
\hline & 2 & 3 & 571232 & 9908572 \\
\hline & 2 & 4 & 571283 & 9908462 \\
\hline & 3 & 1 & 571397 & 9908899 \\
\hline & 3 & 2 & 571485 & 9908777 \\
\hline & 3 & 3 & 571392 & 9908805 \\
\hline & 3 & 4 & 571489 & 9908866 \\
\hline & 4 & 1 & 572063 & 9909424 \\
\hline & 4 & 2 & 572176 & 9909440 \\
\hline & 4 & 3 & 572091 & 9909496 \\
\hline & 4 & 4 & 572126 & 9909348 \\
\hline & 5 & 1 & 572090 & 9909564 \\
\hline & 5 & 2 & 572055 & 9909658 \\
\hline & 5 & 3 & 572170 & 9909639 \\
\hline & 5 & 4 & 572103 & 9909714 \\
\hline & 6 & 1 & 572589 & 9910075 \\
\hline & 6 & 2 & 572595 & 9910216 \\
\hline & 6 & 3 & 572535 & 9910132 \\
\hline & 6 & 4 & 572658 & 9910141 \\
\hline \multirow{20}{*}{ Nuevo Belén } & 1 & 1 & 564804 & 9893916 \\
\hline & 1 & 2 & 564947 & 9893929 \\
\hline & 1 & 3 & 564862 & 9893997 \\
\hline & 1 & 4 & 564888 & 9893850 \\
\hline & 2 & 1 & 564785 & 9893940 \\
\hline & 2 & 2 & 564730 & 9894074 \\
\hline & 2 & 3 & 564694 & 9893987 \\
\hline & 2 & 4 & 564819 & 9894032 \\
\hline & 3 & 1 & 564784 & 9894483 \\
\hline & 3 & 2 & 564696 & 9894584 \\
\hline & 3 & 3 & 564685 & 9894495 \\
\hline & 3 & 4 & 564791 & 9894570 \\
\hline & 4 & 1 & 564967 & 9894447 \\
\hline & 4 & 2 & 564973 & 9894538 \\
\hline & 4 & 3 & 565083 & 9894514 \\
\hline & 4 & 4 & 565063 & 9894411 \\
\hline & 5 & 1 & 564389 & 9894235 \\
\hline & 5 & 2 & 564364 & 9894373 \\
\hline & 5 & 3 & 564310 & 9894279 \\
\hline & 5 & 4 & 564452 & 9894312 \\
\hline
\end{tabular}


diferenciar el sexo de las flores en masculinas, femeninas o hermafroditas. Cuando las palmeras no registraron la presencia de flores, frutos o ambos, y no se encuentran evidencias de la presencia de vestigios de inflorescencias antiguas, semillas, frutos o grupo de plántulas germinadas bajo el árbol adulto se considera con sexo indeterminado. En el caso de ungurahui por ser una planta monoica no se consideró el sexo de las plantas.

\section{Análisis de la información}

La densidad poblacional de las dos especies se obtuvo mediante el cociente entre el número de individuos y el área de muestreo, para ello se aplicó la siguiente fórmula: $D=N / A$. Donde $\mathrm{D}=$ Densidad poblacional, $\mathrm{N}=$ Número de individuos de la especie y $\mathrm{A}=$ Superficie del área de levantamiento. Este mismo procedimiento se usó para el cálculo por clases diamétricas y alturas. Los cálculos también se hicieron para plantas según estado fenológico y el número de racimos, para el aguaje se consideró el cálculo de plantas según sexo.

\section{RESULTADOS}

\section{Densidad de Mauritia flexuosa y Oeno- carpus bataua}

La densidad del aguaje y ungurahui adultos y según categorías de tamaño en las tres comunidades se muestra en la Tabla 2. La densidad total del aguaje incluidas todas las categorías de regeneración es de 105,7 ind./ha con un total de 317 individuos en las tres parcelas de muestreo en Nueva Jerusalén, mientras que Nuevo Belén presenta una densidad de 85 ind./ha con un total de 425 individuos en las 5 parcelas. El número de plantas de la categoría adulta es mayor en Nuevo Belén con 67,8 ind./ha y un total de 339 individuos en las cinco parcelas de muestreo, mientras que Nueva Jerusalén presenta 45,3 ind./ha y un total de 136 individuos en las 3 hectáreas de muestreo.

Las categorías de regeneración se distribuyen de la siguiente manera: en Nueva Jerusalén los latizales suman 181,0 y 60,3 ind./ha, superando a los adultos, mientras que los brinzales están ausentes en el bosque. En la comunidad de Nuevo Belén los latizales y brinzales suman en total 61,0 y 25,0 individuos con 12,2 y 5,0 ind./ha correspondientemente.

La abundancia de ungurahui se distribuye de la siguiente manera: la zona de Mashunta muestra una mayor cantidad con un total de 153 individuos de todas las categorías de tamaño en las seis parcelas y $25,5 \mathrm{ind} / \mathrm{ha}$. En la comunidad de Nueva Jerusalén se encontraron un total de 82 individuos de todas las categorías de tamaño en las tres parcelas y 27,3 ind./ha, mientras que los individuos adultos suman en total 60,0 y 20,0 por ha. Los latizales y brinzales de ungurahui por hectárea se presentan en cantidades muy bajas en ambas áreas, en Nuevo Jerusalén existen 4,3 latizales y 3,0 brinzales y en Mashunta 4,8 latizales y 4,2 brinzales por hectárea.

\section{Distribuciones diamétricas y por altu- ra}

La distribución del número de plantas por clases diamétricas para el aguaje muestra una forma de campana con una mayor concentración de individuos con mayor diámetro en las categorías entre $21-30$ y $31-40 \mathrm{~cm}$ (Figura 2). Los individuos de ungurahui no desarrollan mucho diámetro, solamente están presentes en dos categorías diamétricas, entre 10-20 donde son más escasos con 9 ind./ha y 5 ind./ha en Nueva Jerusalén y Mashunta respectivamente, y más abundantes entre $20-30 \mathrm{~cm}$ con 11 ind./ha en ambas áreas (Figura 3).

En las Figuras 4 y 5 se muestra la distribución del número de individuos por clases de altura con un rango de $5 \mathrm{~m}$, la distribución que presentan tiene forma de campana en las dos especies en ambas comunidades. Para las dos especies, las alturas máximas reportadas alcanzan los $30 \mathrm{~m}$, de igual modo las clases de altura más representativas están entre los 16 y $20 \mathrm{~m}$ y entre los 21-25 m, es probable que las clases entre los 26 y $30 \mathrm{~m}$ y entre los 31-35 m presentan menores cantidades debido a la intervención que sufrieron estos bosques al cortar las palmeras de estas categorías con el fin de cosechar los frutos. 
Tabla 2. Densidad de M. flexuosa y Oenocarpus bataua por categorías de regeneración en dos comunidades de la cuenca alta del Putumayo.

\begin{tabular}{|c|c|c|c|c|c|c|c|c|c|}
\hline \multirow{2}{*}{ Comunidad } & \multirow{2}{*}{ Parcela } & \multicolumn{3}{|c|}{ Mauritia flexuosa (ind./ha) } & \multirow{2}{*}{ TOTAL } & \multicolumn{3}{|c|}{ Oenocarpus bataua (ind./ha) } & \multirow{2}{*}{ TOTAL } \\
\hline & & Adulto & Latizal & Brinzal & & Adulto & Latizal & Brinzal & \\
\hline \multirow{3}{*}{ Nueva Jerusalén } & 1 & 43,0 & 67,0 & 0,0 & 110,0 & 5,0 & 1,0 & 0,0 & 6,0 \\
\hline & 2 & 34,0 & 6,0 & 0,0 & 40,0 & 23,0 & 8,0 & 6,0 & 37,0 \\
\hline & 3 & 59,0 & 108,0 & 0,0 & 167,0 & 32,0 & 4,0 & 3,0 & 39,0 \\
\hline Promedio & & 45,3 & 60,3 & 0,0 & 105,7 & 20,0 & 4,3 & 3,0 & 27,3 \\
\hline \multicolumn{2}{|l|}{ Subtotal } & 136,0 & 181,0 & 0,0 & 317,0 & 60,0 & 13,0 & 9,0 & 82,0 \\
\hline \multirow{5}{*}{ Nuevo Belén } & 1 & 86,0 & 10,0 & 0,0 & 96,0 & & & & \\
\hline & 2 & 35,0 & 1,0 & 2,0 & 38,0 & & & & \\
\hline & 3 & 98,0 & 40,0 & 13,0 & 151,0 & & & & \\
\hline & 4 & 60,0 & 7,0 & 8,0 & 75,0 & & & & \\
\hline & 5 & 60,0 & 3,0 & 2,0 & 65,0 & & & & \\
\hline Promedio & & 72,7 & 16,7 & 7,7 & 97,0 & & & & \\
\hline Subtotal & & 339,0 & 61,0 & 25,0 & 425,0 & & & & \\
\hline \multirow{6}{*}{ Mashunta } & 1 & & & & & 10,0 & 0,0 & 0,0 & 10,0 \\
\hline & 2 & & & & & 10,0 & 4,0 & 1,0 & 15,0 \\
\hline & 3 & & & & & 5,0 & 6,0 & 2,0 & 13,0 \\
\hline & 4 & & & & & 15,0 & 4,0 & 6,0 & 25,0 \\
\hline & 5 & & & & & 35,0 & 10,0 & 8,0 & 53,0 \\
\hline & 6 & & & & & 24,0 & 5,0 & 8,0 & 37,0 \\
\hline Promedio & & & & & & 24,7 & 6,3 & 7,3 & 38,3 \\
\hline Subtotal & & & & & & 99,0 & 29,0 & 25,0 & 153,0 \\
\hline Promedio general & & 59,0 & 38,5 & 3,8 & & 22,3 & 5,3 & 5,2 & 32,8 \\
\hline Total & & 475,0 & 242,0 & 25,0 & & 159,0 & 42,0 & 34,0 & 235,0 \\
\hline
\end{tabular}

Estado fenológico y producción de racimos

Del total de plantas de aguaje adultas en la comunidad de Nueva Jerusalén, el $60 \%$ de ellas se encuentran en estado fértil alcanzando 27 ind./ha, mientras que en Nuevo Belén el $87 \%$ están fértiles representando el 58,8 ind./ha. Así mismo, del total de plantas fértiles solamente el $65,4 \%$ y $21,1 \%$ de plantas se encuentran en la fase fenológica de espádice en Nueva Jerusalén y Nuevo Belén, correspondientemente. Existe un alto número de plantas fértiles en estado fenológico con inflorescencias, $78,9 \%$ en Nuevo Belén y solamente el $29,6 \%$ en Nuevo Jerusalén (Tabla 3).

En ambas áreas, la producción de racimos es de 3 por planta, existe un alto número de racimos en Nuevo Belén con 174 por hectárea mientras que en Nueva Jerusalén es menor con 77 racimos por hectárea.

En el caso del ungurahui, las dos áreas de estudio presentan un promedio de 12 plantas/ha en estado fértil, de igual modo también presentan igual cantidad de plantas con infrutescencias, 9 ind./ha. La producción a nivel de número de racimos es superior en Nuevo Jerusalén con un total de 72 y 24 racimos/ha, mientras que Mashunta, presenta un total de 90 individuos y una densidad de 15 racimos/ha. Realizando un cálculo según número de sacos producidos (1 racimo de igual a 0,5 saco) el área de Nuevo Jerusalén estaría produciendo aproximadamente 12 sacos por hectárea de ungurahui (Tabla 4). 
Tabla 3. Número de individuos según estado fenológico de aguaje y ungurahui durante el mes de setiembre del año 2019 en dos comunidades de la cuenca alta del Putumayo.

\begin{tabular}{|c|c|c|c|c|c|c|}
\hline \multirow{2}{*}{ Especie } & \multirow{2}{*}{ Comunidad } & \multicolumn{3}{|c|}{ Número de plantas } & \multirow{2}{*}{$\begin{array}{l}\text { Plantas } \\
\text { fértiles }\end{array}$} & \multirow{2}{*}{$\begin{array}{l}\mathbf{N}^{\circ} \\
\text { racimos }\end{array}$} \\
\hline & & Espádice & Inflorescencia & Infrutescencia & & \\
\hline \multirow{2}{*}{ Aguaje } & Nueva Jerusalén & 53 & 24 & 4 & 81 & 232 \\
\hline & Nuevo Belén & 62 & 232 & 1 & 295 & 872 \\
\hline \multirow{2}{*}{ Ungurahui } & Nueva Jerusalén & 2 & 5 & 28 & 35 & 72 \\
\hline & Mashunta & 16 & 0 & 56 & 72 & 90 \\
\hline
\end{tabular}

Tabla 4. Comparación del porcentaje de plantas de Mauritia flexuosa L.f. (aguaje) durante el mes de setiembre del año 2019 en aguajales de dos comunidades de la cuenca del Putumayo.

\begin{tabular}{|c|c|c|c|c|c|}
\hline \multirow[b]{2}{*}{ Comunidad } & \multirow[b]{2}{*}{ Parcela } & \multicolumn{3}{|c|}{ Estado fenológico } & \multirow[b]{2}{*}{$\%$ Total } \\
\hline & & $\begin{array}{c}\text { Adulto } \\
\text { indeterminado }\end{array}$ & Adulto masculino & $\begin{array}{c}\text { Adulto } \\
\text { femenino }\end{array}$ & \\
\hline \multirow{3}{*}{ Nueva Jerusalén } & 1 & 2,0 & 63,0 & 35,0 & 100,0 \\
\hline & 2 & 3,0 & 79,0 & 18,0 & 100,0 \\
\hline & 3 & 5,0 & 66,0 & 29,0 & 100,0 \\
\hline \multirow[t]{3}{*}{ Promedio } & & 3,3 & 69,3 & 27,4 & 100,0 \\
\hline & 1 & 6,0 & 71,0 & 23,0 & 100,0 \\
\hline & 2 & 3,0 & 77,0 & 20,0 & 100,0 \\
\hline \multirow[t]{3}{*}{ Nuevo Belén } & 3 & 2,0 & 61,0 & 37,0 & 100,0 \\
\hline & 4 & 0,0 & 60,0 & 40,0 & 100,0 \\
\hline & 5 & 1,0 & 67,0 & 32,0 & 100,0 \\
\hline Promedio & & 2,4 & 67,2 & 30,4 & 100,0 \\
\hline Promedio general & & 2,9 & 68,3 & 28,9 & 100,0 \\
\hline
\end{tabular}

\section{Relación porcentual del sexo del aguaje}

La distribución porcentual del sexo de las palmeras de aguaje adultas en los aguajales es de gran importancia teniendo en consideración que estas son las que producen los frutos, por lo tanto esto nos permite calcular la producción y el estado del aguajal en términos de uso y forma de intervención (destructivo o con manejo) en años anteriores. Los aguajales en estudio de las zonas de Nuevo Belén y Nueva Jerusalén presentan un mayor porcentaje de plantas adultas masculinas con $67,0 \%$ y $69,0 \%$ correspondientemente. 


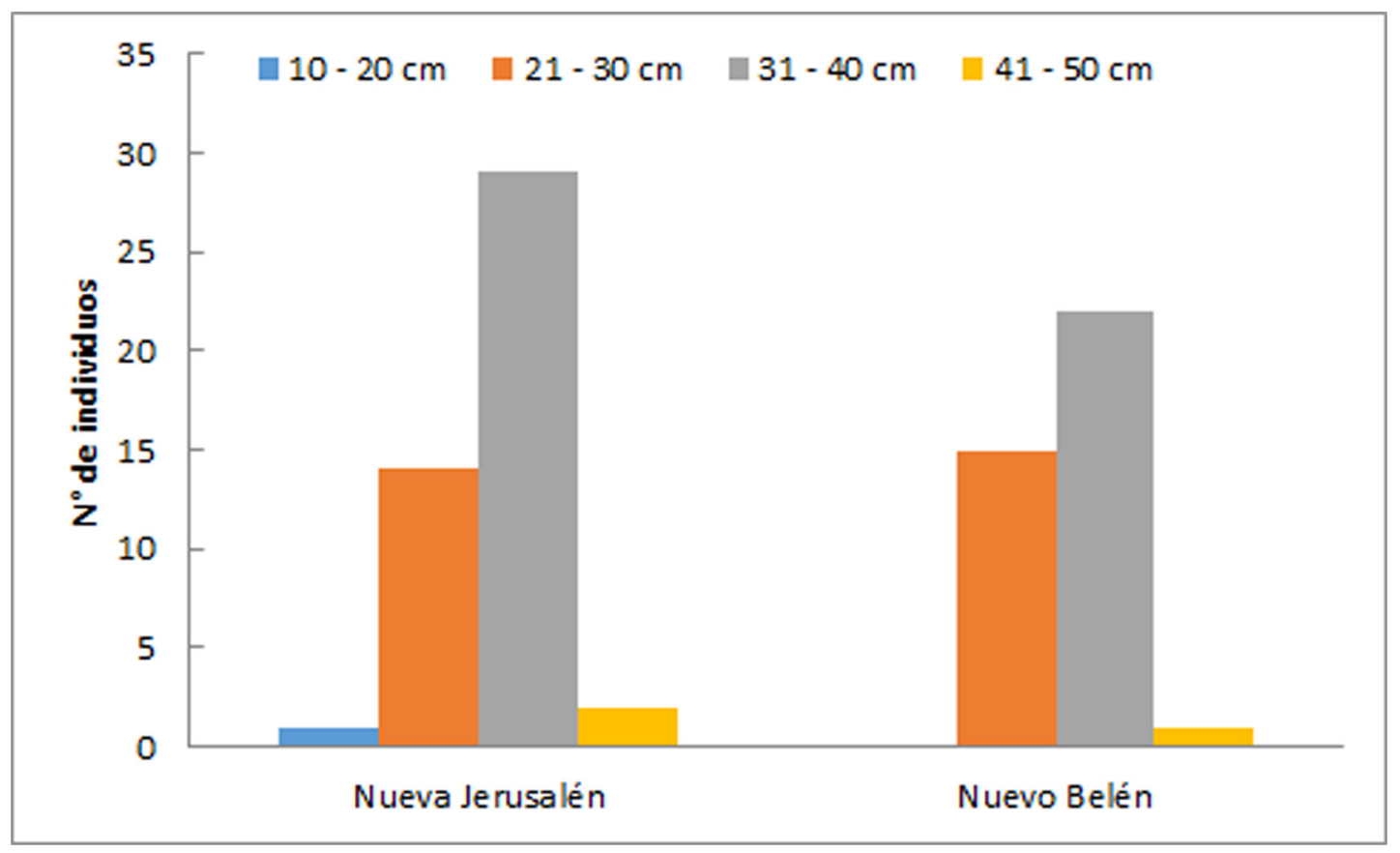

Figura 2. Distribución del número de plantas/ha y clase diamétrica de aguaje en dos comunidades de la cuenca del Putumayo.

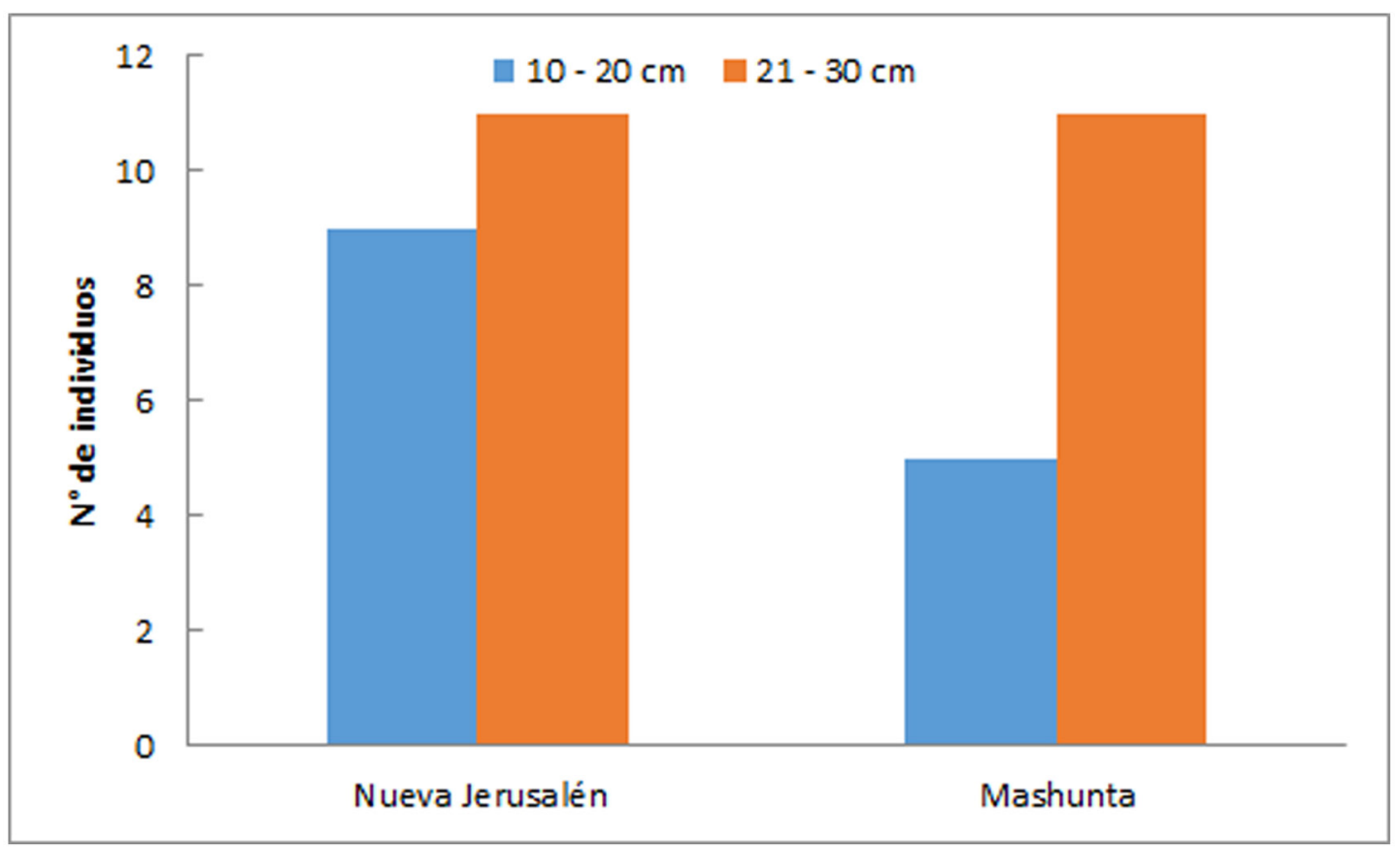

Figura 3. Distribución del número de plantas/ha y clases diamétricas de ungurahui en dos comunidades de la cuenca del Putumayo. 


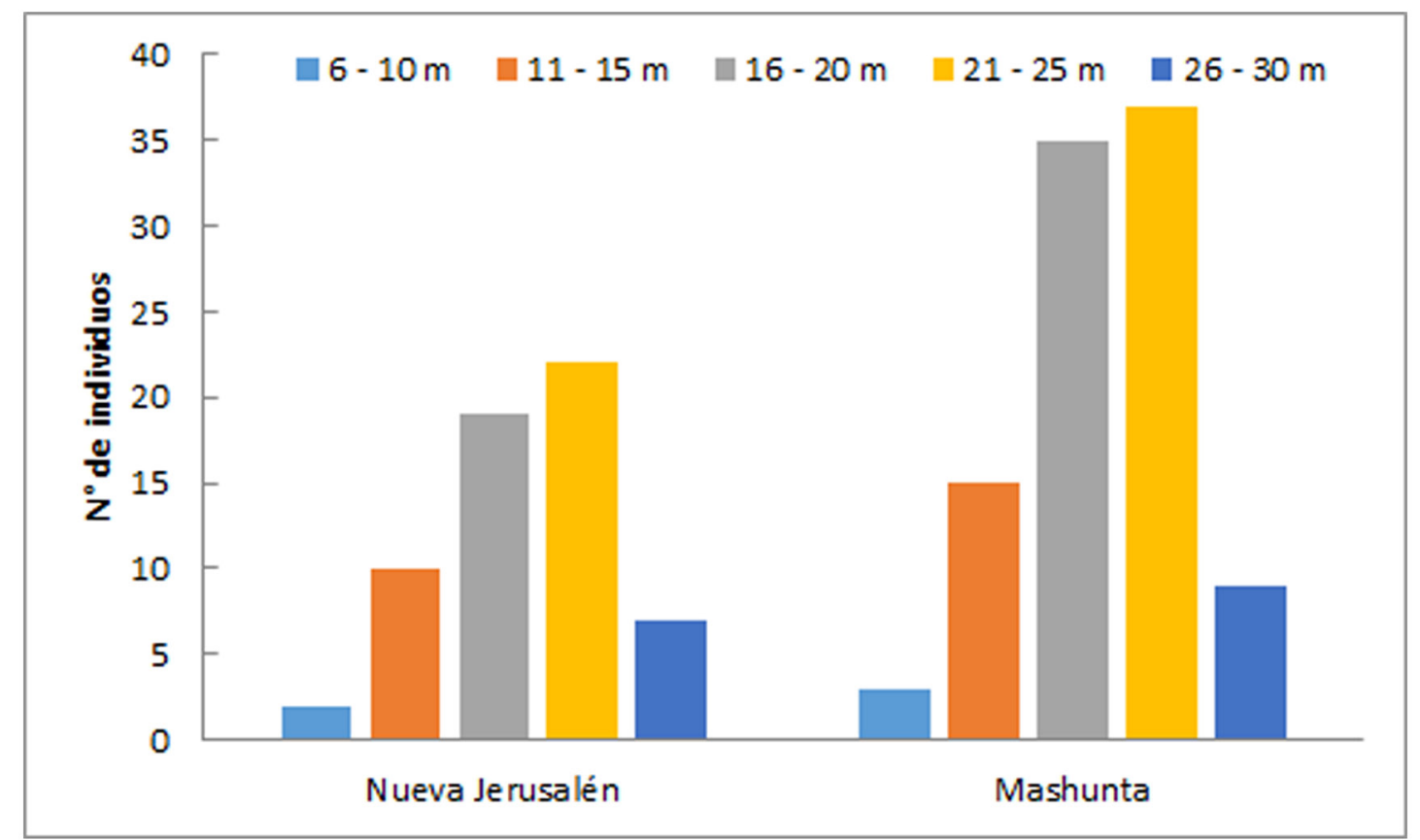

Figura 4. Distribución del número de individuos de aguaje por clases de altura en dos comunidades de la cuenca del Putumayo.

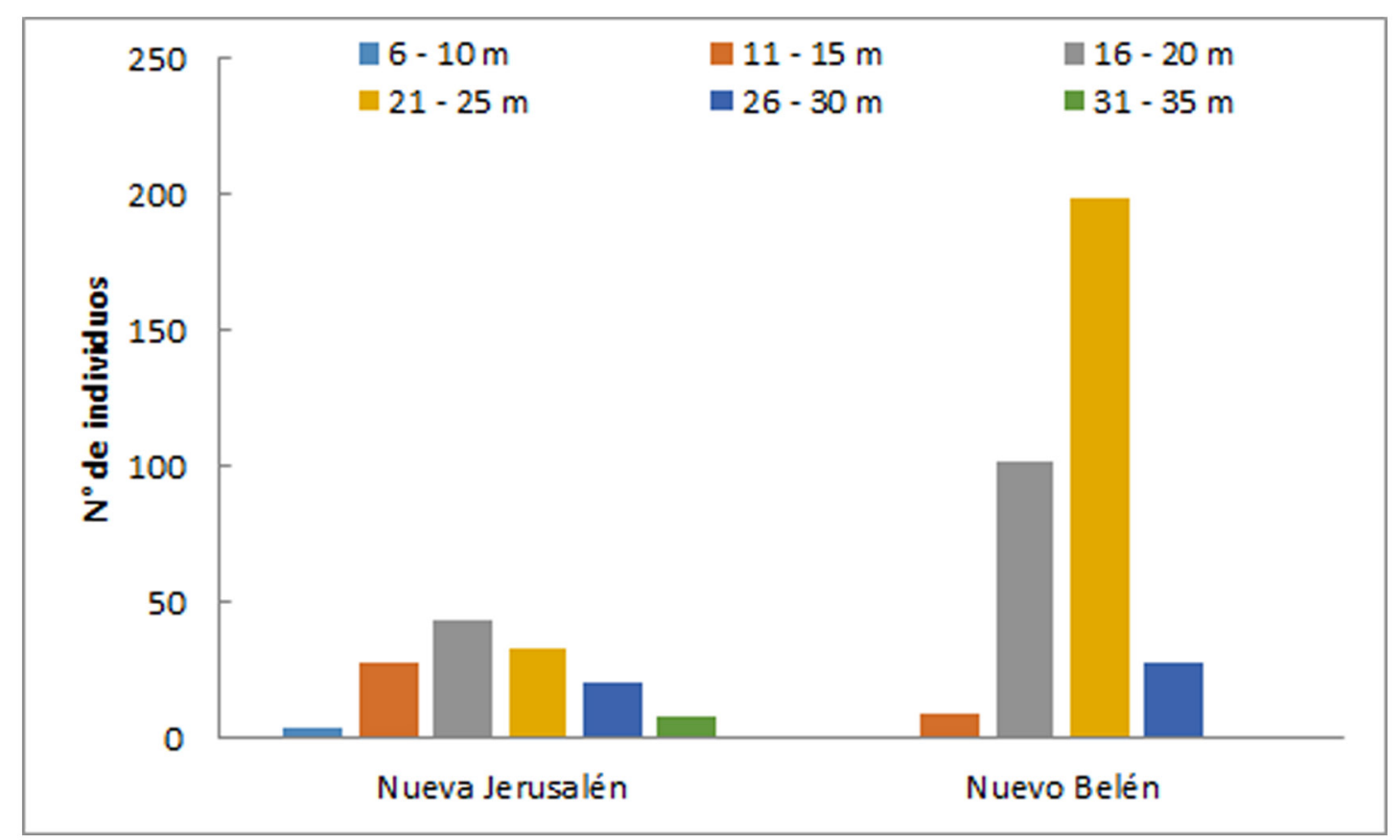

Figura 5. Distribución del número de individuos de ungurahui por clases de altura en dos comunidades de la cuenca del Putumayo. 


\section{DISCUSIÓN}

La densidad en estos aguajales es bastante baja, variando entre 45,3 en Nueva Jerusalén y 67,8 en Nuevo Belén. En otras zonas de la Amazonía peruana hay mayor densidad y superan los 250 individuos por hectárea, y los que presentan menores densidades son considerados como aguajales semidensos o mixtos (García-Soria et al., 2012; y Zárate et al., 2013). Los aguajales de la zona del río Marañón en la Reserva Nacional Pacaya Samiria presentan densidades superiores a los 200 individuos por hectárea (Freitas y Flores, 2015; Freitas, 1996; Gonzales et al., 2007; Kahn y Mejía, 1990); mientras que Bejar (2014) y Buendía (2016) reportan cantidades entre 35 a 50 aguajes adultos por hectárea en estudios realizados en Pucallpa, región Ucayali y en la Reserva Allpahuayo-Mishana en la región Loreto; resultados similares a los encontrados en esta investigación.

Sin lugar a dudas estos aguajales pueden ser considerados de muy baja densidad ya que han sido aprovechados mediante la tala, práctica común empleada por los pobladores de estas comunidades, razón por la cual pueden llegar a presentar niveles tan bajos de abundancia por hectárea. Resultados similares al presente estudio son reportados por Freitas (2012), quien manifiesta que en aguajales sometidos a fuerte aprovechamiento la abundancia del número de individuos es de 65 ( \pm 10 ) individuos por hectárea y en aguajales con mediano aprovechamiento de 77 ( \pm 18 ) individuos por hectárea.

Las categorías de regeneración también presentan densidades muy bajas; es importante contar con una mayor cantidad de latizales, pues este grupo asegura su permanencia en el bosque, sin embargo consideramos que las cantidades por hectárea que presentan estos bosques, de 12,2 en Nuevo Belén a 60,3 en Nueva Jerusalén, son muy bajas si comparamos la regeneración en aguajales con bajo aprovechamiento (menos de 5 individuos talados por ha) los cuales presenta 556 latizales y 223 brinzales por hectárea, pero similares a aguajales con fuerte aprovechamiento quiénes presentan 30,6 latizales y 10 brinzales por hectárea (Freitas, 2012).

Al igual que en el aguaje, el ungurahui registra bajas densidades, nuestros resultados son comparables con los resultados obtenidos por Dávila (2015) y Góngora (2018) quiénes repor$\tan$ entre 18 a 34 individuos por hectárea en los bosques de la Reserva Nacional Allpahuayo Mishana, los que a pesar de encontrarse en un área protegida fueron intervenidas en años anteriores por encontrarse cercanos a centros poblados. Los bosques en estudio presentan una abundancia significativamente inferior a la de un bosque sub andino poco intervenido en Bolivia, con 198 individuos por hectárea (Miller, 2002).

La cantidad de regeneración es mucho más crítica para el caso del ungurahui variando de 4 a 5 latizales por hectárea en las dos comunidades, lo cual podría atribuirse a que en estos bosques se han aprovechado los individuos productivos talando la palmera, practica muy común realizado por los pobladores de la zona o que la especie muestra una baja capacidad de regeneración.

La distribución del número de árboles por clase diamétrica y por altura es típica de los ecosistemas de aguajal el cual difiere de la distribución de especies latifoliadas en bosques primarios donde la distribución asemeja a una J invertida (Louman et al., 2001). La forma acampanada de la curva del aguaje se explica por su limitado crecimiento lateral como palmera (Freitas, 2012), generalmente los individuos jóvenes de aguaje tienen un estípite grueso que mantiene hasta a su madurez; es así que muchos individuos se concentran en la categoría de 30-40 $\mathrm{cm}$ de diámetro. En el caso de los árboles existe una relación entre el diámetro y la altura, esto no ocurre con el aguaje; Goodman et al. (2013) mencionan que, la altura de la palmera es la variable que mejor estima su biomasa.

El aguajal de Nuevo Belén presenta una baja producción de racimos, alcanzando 174 y 59 
plantas por hectárea, mientras que en Nuevo Jerusalén es de 77 racimos por hectárea y 27 plantas en producción. Haciendo cálculos productivos, podríamos obtener en el caso de Nuevo Belén 130 sacos (unidad de medida de volumen de cosecha) de frutos de aguaje por hectárea (1 racimo es igual a 0,75 sacos). Nuestros resultados difieren a lo reportado en la comunidad de Veinte de Enero, Reserva Nacional Pacaya Samiria, por Gonzales et al., (2007) quienes mencionan que en esos aguajales existen un promedio de 30 plantas hembras aprovechables por hectárea, 138 racimos por hectárea con una producción máxima anual de 103 sacos por hectárea.

En ambas zonas, predominan los individuos masculinos que va entre 67 y $69 \%$, es un indicativo de que estos aguajales han sido aprovechadas mediante la tala de palmeras femeninas (o hermafroditas), lo cual es evidente debido a que en los trabajos de campo se observaron restos de individuos cortados. Nuestros resultados son similares a los reportados por Freitas (2012), indicando que los aguajales aprovechados mediante métodos destructivos con fuerte intensidad presentan una proporción de sexos con $82,6 \%$ de masculinos y $17,4 \%$ de femeninos, mientras que en los aguajales sin intervención es de $51 \%$ femeninas (o hermafroditas) y $49 \%$ de masculinas (Urrego, 1987).

Los aguajales de las dos comunidades actualmente vienen siendo aprovechadas con poca aplicación de técnicas de manejo, como el escalamiento de los árboles (Manzy y Coomes, 2009), esta acción viene repercutiendo en el abastecimiento de frutos para consumo de la población, y puede ser más problemático si tenemos en consideración que los principales animales de caza que consume la población utilizan un alto porcentaje del fruto en sus alimentación (Endress et al., 2013), por lo tanto se estaría afectando indirectamente a este grupo de animales (Pérez-Peña et al., 2016). El paso de una actividad menos sostenible a una más sostenible implicaría hacer uso de las técnicas de escalamiento, acceso a mejor precio de mercado y mejorar la organización comunal (Horn et al., 2012; Schulz et al., 2019), es decir el manejo de este recurso debe tener un enfoque multidisciplinario (Virapongse et al., 2017) con la finalidad de tener un mismo horizonte en la conservación de este recurso y mejoramiento económico de las comunidades nativas.

\section{CONCLUSIONES}

La densidad de los bosques de aguaje y ungurahui es baja comparada con bosques no intervenidos. En el caso del aguaje esta situación es atribuida principalmente al sistema de aprovechamiento practicado por los pobladores de la zona que mayormente cortan las palmeras adultas femeninas (o hermafroditas) para el aprovechamiento de los frutos, lo cual determina la dominancia de individuos masculinos y por lo tanto una baja producción de frutos.

Este estudio nos permite conocer la estructura y el estado de intervención en las que se encuentran estos bosques donde predominan dos frutales de importancia social y ecológica en las comunidades de Nueva Jerusalén, Nuevo Belén y Mashunta. La información básica nos permite orientar a tomar medidas correctivas para el manejo sostenible de estos ecosistemas. Mashunta, Nuevo Jerusalén y Nuevo Belén son comunidades indígenas cuya ingesta de proteína animal está basado en mamíferos que consumen principalmente frutos de aguaje y ungurahui, un mayor deterioro de sus bosques significaría un mayor deterioro de sus calidad de vida. Se recomienda campañas de largo plazo de educación ambiental orientadas a la enseñanza del uso de subidores para el aprovechamiento y manejo sostenible de los frutos evitando el corte de aguaje y ungurahui.

\section{AGRADECIMIENTO}

A los pobladores de las comunidades nativas de Nueva Jerusalén, Mashunta y Nuevo Belén por su hospitalidad y apoyo en los trabajos de campo. Al Fondo Nacional de Desarrollo Científico, Tecnológico y de Innovación Tecnológi- 
ca-FONDECYT, por el financiamiento del proyecto "Animales de caza y palmeras nativas en la seguridad alimenticia y en la lucha contra la pobreza económica en comunidades indígenas de la cuenca alta del río Putumayo, frontera Perú-Colombia", Contrato N 136-2018-FONDECYT-BM-IADT-AV.

\section{REFERENCIAS BIBLIOGRÁFICAS}

Aquino, R. (2005) Alimentación de mamíferos de caza en los «aguajales» de la Reserva Nacional de Pacaya-Samiria (Iquitos, Perú). Revista Peruana de Biología, 12(3), 417-425. http://dx.doi.org/10.15381/rpb. v26i2.16378

Acevedo, J. F. y Zamora, J. G. (2016) Papel de los mamíferos en los procesos de dispersión y depredación de semillas de Mauritia flexuosa (Arecaceae) en la Amazonía colombiana. Revista de Biología Tropical, 64(1), 5-15. 10.5517/RBT.V64I1.18157

Balslev, H., Grandez, C., Paniagua, N. Y., Møller, A. L. y Hansen, S. L. (2008) Palmas (Arecaceae) útiles en los alrededores de Iquitos, Amazonía Peruana. Revista Peruana de Biología, 15, 121-132. http://dx.doi. org/10.15381/rpb.v26i2.16378

Beck, H. (2006) A Review of Peccary-Palm Interactions and Their Ecological Ramifications across the Neotropics, Journal of Mammalogy, 87(3), 519-530, https://doi. org/10.1644/05-MAMM-A-174R1.1

Bejar, A. M. (2014) Evaluación de Rodales Naturales de Aguaje "Mauritia flexuosa L.f" con fines de Aprovechamiento Sostenido en la Comunidad Cametsa Quipatsi - Suaya en la Región Ucayali (Tesis de pregrado). Universidad Nacional de la Amazonía Peruana. Iquitos.

Bernal, R. y Galeano, G. (2013) Cosechar sin destruir - Aprovechamiento sostenible de palmas colombianas. Bogotá: Universidad Nacional de Colombia.

Bodmer, R. E., Puertas, P. E., Garcia, J. E., Dias, D. R. y Reyes, C. (1999) Game animals, palms, and people of the flooded forests: Management considerations for the Pa-
caya-Samiria national reserve, Peru. Advances in Economic Botany, 13, 217-231. https://www.jstor.org/stable/43919749

Bodmer, R. E. (1991) Strategies of seed dispersal and seed predation in Amazonian ungulates. Biotropica, 3(3),255-261. https:// doi.org/10.2307/2388202

Buendía, B. (2016) Evaluación del potencial de rodales naturales de aguaje "Mauritia flexuosa L.f." con fines de aprovechamiento del fruto en la Reserva Nacional Allpahuayo Mishana, Loreto, Perú (Tesis de pregrado). Universidad Nacional de la Amazonía Peruana. Iquitos.

Dávila, V. A. (2015) Relación altura y diámetro con la productividad de frutos de Oenocarpus bataua Mart «ungurahui», distrito San Juan Bautista, Loreto, Perú-2014. (Tesis de pregrado). Universidad Nacional de la Amazonía Peruana. Iquitos.

Draper, F. C., Roucoux, K. H., Lawson, I. T., Mitchard, E. T., Coronado, E. N. H., Lähteenoja, O., Montenegro, L., Valderrama E., Zarate R. y Baker, T. R. (2014) The distribution and amount of carbon in the largest peatland complex in Amazonia. Environmental Research Letters, 9(12), 124017. doi:10.1088/1748-9326/9/12/124017

Encarnación, F. (1993) El bosque y las formaciones vegetales en la llanura amazónica del Perú. Alma Mater, 6, 95-114.

Endress, B. A., Horn, C. M. y Gilmore, M. P. (2013) Mauritia flexuosa palm swamps: composition, structure and implications for conservation and management. Forest ecology and management, 302, 346-353.

Freitas, L. (1996) Caracterización florística y estructural de cuatro comunidades boscosas de la llanura aluvial inundable en la zona de Jenaro Herrera, Amazonía Peruana. Iquitos: Instituto de Investigaciones de la Amazonía Peruana.

Freitas, L. (2012) Impacto del aprovechamiento en la estructura, producción y valor de uso del aguaje en la Amazonía peruana. Recursos Naturales y Ambiente 67, 38-47.

Freitas, L., Otárola, E., Del Castillo, D., Linares, C., Martínez, P. y Malca, G. (2006) Servicios ambientales de almacenamiento y secuestro de carbono del ecosistema aguajal 
en la Reserva Nacional Pacaya Samiria, Loreto-Perú. Iquitos: Instituto de Investigaciones de la Amazonía Peruana.

Freitas, L., Pinedo, M., Linares, C. y Del CastiIlo, D. (2006) Descriptores para el aguaje (Mauritia flexuosa $L F$ ). Iquitos: Instituto de Investigaciones de la Amazonía Peruana.

Freitas, L. y Flores, H. (2015) Condición silvicultural de la palmera Mauritia flexuosa Lf en el ecosistema "Aguajal" de Parinari, Loreto, Perú. Folia Amazónica, 24 (2), 155-162. https://doi.org/10.24841/fa.v12i1-2.123.

García-Soria, D., Honorio-Coronado, E. N. y del Castillo-Torres, D. (2012) Determinación del stock de carbono en aguajales de la cuenca del río Aguaytía, Ucayali-Perú. Folia Amazónica, 21(1-2), 153-160.

Gilmore, M. P., Endress, B. A. y Horn, C. M. (2013) The socio-cultural importance of Mauritia flexuosa palm swamps (aguajales) and implications for multi-use management in two Maijuna communities of the Peruvian Amazon. Journal of Ethnobiology and Ethnomedicine, 9, 29. https://doi. org/10.1186/1746-4269-9-29.

Góngora, D. A. (2018) Asociación entre altura y diámetro en las plantas de Oenocarpus bataua Mart «Ungurahui». Distrito San Juan Bautista, Loreto, Perú-2014. (Tesis de pregrado). Universidad Nacional de la Amazonía Peruana. Iquitos.

Gonzales, D. E., Noriega, P. R., Llanos, J. D., Paredes, J. Z. y Paredes, J. M. (2007) Plan de Manejo Forestal de Mauritia flexuosa en la Comunidad Veinte de Enero, cuenca Yanayacu Pucate-Reserva Nacional Pacaya Samiria 2005-2007. Iquitos: Fundación ProNaturaleza-Agencia de Desarrollo Internacional de los Estados Unidos-The Nature Conservancy.

Goodman, R. C., Phillips, O. L., Del Castillo, D., Freitas, L., Tapia, S., Monteagudo, A. y Baker, T. R. (2013) Amazon palm biomass and allometry. Forest Ecology and Management, 310, 994-1004. https://doi. org/10.1016/j.foreco.2008.09.038.

Hergoualc'h, K., Gutiérrez-Vélez, V. H., Menton, M. y Verchot, L. V. (2017) Characterizing degradation of palm swamp peatlands from space and on the ground: an exploratory study in the Peruvian Amazon. Forest Ecology and Management, 393, 63-73.

Honorio, E. N., Pennington, T. R., Freitas, L. A., Nebel, G. y Baker, T. R. (2008) Análisis de la composición florística de los bosques de Jenaro Herrera, Loreto, Perú. Revista Peruana de Biología, 15(1), 53-60.

Horn, C. M., Gilmore, M. P., Vargas, V. H. y Endress, B. A. (2018) Spatio-temporal patterns of Mauritia flexuosa fruit extraction in the Peruvian Amazon: Implications for conservation and sustainability. Forest Ecology and management, 267, 93-103.

Horn, C. M., Gilmore, M. P., y Endress, B. A. (2012) Ecological and socio-economic factors influencing aguaje (Mauritia flexuosa) resource management in two indigenous communities in the Peruvian Amazon. Forest Ecology and Management, 267, 93103.

Horna, L. Y. F. y Coronado, E. N. H. (2018) Evaluación de las técnicas de aprovechamiento de frutos de aguaje (Mauritia flexuosa Lf) en el distrito de Jenaro Herrera, Loreto, Perú. Folia Amazónica, 27(2), 131-150.

Jarrín, P. S., Tapia Carrillo, L., \& Zamora, G. (2017) Demografía y transformación territorial: medio siglo de cambio en la región amazónica de Ecuador. Eutopia, 12 81-100.

Kahn, F. y Mejía, K. (1990) Palm communities in wetland forest ecosystem of Peruvian Amazonia. Forest Ecology and management, 33-34, 169-179. 10.1016/j.foreco.2011.11.040.

Lähteenoja, O. y Page, S. (2011) High diversity of tropical peatland ecosystem types in the Pastaza-Marañón basin, Peruvian Amazonia. Journal of Geophysical Research: Biogeosciences, 116, G02025. 10.1029/2010JG001508.

Listabarth, C. (1999) The palms of the Surumoni area (Amazonas, Venezuela). II. Phenology and pollination of two flooded forest palms, Mauritiella aculeata and Leopoldinia pulchra. Acta Botanica Venezuelica, 22(1), 153-165.

López-Parodi, J. y Freitas, D. (1990) Geographical aspects of forested wetlands in the 
lower Ucayali, Peruvian Amazonia. Forest Ecology and management, 33-34, 157168. 10.1016/j.foreco.2011.11.040.

Louman, B., Quiróz, D. y Nilsson, M. (2001) Silvicultura de bosques latifoliados húmedos con énfasis en América Central. Turrialba: CATIE.

Manzi, M., y Coomes, O. T. (2009) Managing Amazonian palms for community use: a case of aguaje palm (Mauritia flexuosa) in Peru. Forest Ecology and Management, 257(2), 510-517.

Miller, K. (2002) Fruit production of the Ungurahua palm (Oenocarpus bataua Mart. subsp. bataua, Arecaceae) in an indigenous managed reserve. Economic Botany, 56(2), 165-176.

Montúfar, R., Laffargue, A., Pintaud, J. C., Hamon, S., Avallone, S. y Dussert, S. (2010) Oenocarpus bataua Mart. (Arecaceae): Rediscovering a source of high oleic vegetable oil from Amazonia. Journal of the American Oil Chemists' Society, 87(2), 167-172.

Moreira, D. F. y Colombier, M. R. (2019) Mi casa pequeña, mi corazón grande. Política territorial y cosmológica del pueblo Kukama. Mundo Amazónico, 10(1): 157-184.

Neiff, J. J., Casco, S. L. y Arias, C. J. (2004) Glosario de humedales de Iberoamérica. En: Humedales de Iberoamérica. Ed. por Neiff, J.J. La Habana: 336-380.

OSINFOR (Organismo de Supervisión de los Recursos Forestales y de Fauna). (2011) Evaluación de las áreas deforestadas y humedales en los departamentos de Loreto, Ucayali y Madre de Dios al año 2011. Lima: OSINFOR.

Parodi, J. L., y Freitas, D. (1990) Geographical aspects of forested wetlands in the lower Ucayali, Peruvian Amazonia. Forest Ecology and management, 33, 157-168.

PEDICP. (2005) Proyecto especial binacional desarrollo integral de la cuenca del río Putumayo. Compatibilización e integración de la zonificación ecológica-económica del ámbito del PEDICP. Iquitos: PEDICP.

Pérez-Peña, P. E., Gonzales-Tanchiva, C. y Trigoso-Pinedo, M. (2016) Evaluación del plan de manejo de animales de caza en la Re- serva Nacional Pucacuro. Folia Amazónica, 25 (1), 1-16. https://doi.org/10.24841/fa. v12i1-2.123.

Ramos-Rodriguez M. C., Perez-Peña, P. E., Flores-Cardenas G. y Ortiz-Sanchez, A. (2019) Mamiferos. En: Biodiversidad en la cuenca alta del Putumayo, Perú. Ed. por Pérez-Peña, P. E., Ramos-Rodríguez M. C., Díaz-Alván, J., Zarate-Gómez, R. y Mejía-Carhuanca. Iquitos: Instituto de Investigaciones de la Amazonía Peruana (IIAP).

Schulz, C., Brañas, M. M., Pérez, C. N., Villacorta, M. D. A., Laurie, N., Lawson, I. T. y Roucoux, K. H. (2019) Uses, cultural significance, and management of peatlands in the Peruvian Amazon: Implications for conservation. Biological Conservation, 235, 189-198. https://doi.org/10.1016/j. biocon.2019.04.005

Sosnowska, J., Ramirez, D. y Millán, B. (2010) Palmeras usadas por los indígenas Asháninkas en la Amazonía Peruana. Revista Peruana de Biología, 17 (3), 347-352. http:// dx.doi.org/10.15381/rpb.v26i2.16378

Stevenson, P. R., Castellanos, M. C., Pizarro, J. C., y Garavito, M. (2002) Effects of seed dispersal by three ateline monkey species on seed germination at Tinigua National Park, Colombia. International Journal of Primatology, 23(6), 1187-1204. https:// doi.org/10.1023/A:1021118618936

Stevenson, P. R. (2000) Seed dispersal by woolly monkeys (Lagothrix lagothricha) at Tinigua National Park, Colombia: dispersal distance, germination rates, and dispersal quantity. American Journal of Primatology, 50(4), 275-289. https://doi.org/10.1002/ (SICI)1098-2345(200004)50:4<275::AIDAJP4>3.0.CO;2-K

Urrego, L. E. (1987) Estudio preliminares de la fenología de la canangucha (Mauritia flexuosa LF). Colombia amazónica, 2(2), 57-81.

van der Hoek, Y., Solas, S. A., y Peñuela, M. C. (2019) The palm Mauritia flexuosa, a keystone plant resource on multiple fronts. Biodiversity and Conservation, 28(3), 539-551. https://doi.org/10.1007/s10531018-01686-4 
Virapongse, A., Endress, B. A., Gilmore, M. P., Horn, C., y Romulo, C. (2017) Ecology, livelihoods, and management of the Mauritia flexuosa palm in South America. Global Ecology and Conservation, 10, 70-92.

Vormisto, J. (2002) Palms as rainforest resources: how evenly are they distributed in Peruvian Amazonia?. Biodiversity \& Conservation, $11(6), 1025-1045$.

Zárate, R., Mori, T. J. y Maco-García, J. T. (2013) Estructura y composición florística de las comunidades vegetales del ámbito de la carretera Iquitos-Nauta, Loreto-Perú. Folia Amazónica, 22(1-2), 77-89.
Zárate, R., Palacios Vega, J. J., Mejía, K., CoheIlo Huaymacari, G. 2019 Aspectos ecológicos de Mauritia flexuosa y Oenocarpus bataua. En: Biodiversidad en la cuenca alta del Putumayo, Perú. Ed. por Pérez-Peña, P. E., Ramos-Rodríguez M. C., Díaz-Alván, J., Zarate-Gómez, R. y Mejía-Carhuanca. Iquitos: Instituto de Investigaciones de la Amazonía Peruana (IIAP): 62-79.

\section{Conflicto de interés}

Los autores de la presente investigación y publicación del artículo declaramos que no incurrimos en conflictos de intereses. 